\title{
Detecting the use of Intentionally Transmitting Personal Electronic Devices Onboard Commercial Aircraft
}

\author{
Randy Woods \\ Old Dominion University \\ Norfolk, Virginia USA \\ r.s.woods@larc.nasa.gov
}

\author{
Jay J. Ely \\ NASA Langley \\ Hampton, Virginia USA \\ j.j.ely@larc.nasa.gov
}

\author{
Dr. Linda Vahala \\ Old Dominion University \\ Norfolk, Virginia USA \\ lvahala@odu.edu
}

\begin{abstract}
The need to detect unauthorized usage of intentionally transmitting portable electronic devices (PEDs) onboard commercial aircraft is growing, while still allowing passengers to use selected unintentionally transmitting devices, such as laptop computers and CD players during non-critical stages of flight. The following paper presents an installed system for detecting PEDs over multiple frequency bands. Additionally, the advantages of a fixed verses mobile system are discussed. While data is presented to cover the frequency range of $20 \mathrm{MHz}$ to 6.5 $\mathrm{GHz}$, special attention was given to the Cellular/PCS bands as well as Bluetooth and the FRS radio bands. Measurement data from both the semi-anechoic and reverberation chambers are then analyzed and correlated with data collected onboard a commercial aircraft to determine the dominant mode of coupling inside the passenger cabin of the aircraft versus distance from the source. As a final check of system feasibility, several PEDs' transmission signatures were recorded and compared with the expected levels.
\end{abstract}

\section{Introduction}

Cellular telephones and other intentionally radiating devices have the potential to cause interference onboard aircraft by coupling power into sensitive receivers due to the high allowance for spurious radiated emissions into aviation radio frequency bands (up to $-13 \mathrm{dBm}$ )[4]. This coupling of signals can cause interference with critical flight systems and therefore, needs to be detected before causing an anomaly. A second concern is the increased radiating distance cellular/PCS telephones have while airborne could cause disturbances with ground based cellular towers by broadcasting to several towers simultaneously. With the possibility of serious disruptions to systems and there not being a clear necessity for their use, the Federal Communication Commission (FCC) has banned cellular phones for use on airplanes [3], and the Federal Aviation Administration (FAA) prohibits all intentionally transmitting devices unless the aircraft operator has determined non-interference [2].

There are two general approaches for detecting the use of intentionally transmitting devices. First, portable detectors carried by the flight crew, and second an installed system. While a portable system might provide the ability to single out a particular offender, the system will be ineffective during critical stages of flight when the crew must remain seated. Furthermore, information from Alitalia Airlines shows that they were only able to find the offender to within half of the aircraft $22 \%$ of the time and, identify a seat row $8 \%$ of the time with a portable system [1].

In contrast, a permanently installed system will provide continuous uniform coverage of the entire passenger cabin allowing the attendant to transverse the airplane visually searching for the violator when an alarm is received. This will prove to be more valuable in implementation due to the ability to monitor the entire passenger cabin including lavatories.

Leaky coaxial cable is used in many applications such as tunnels and buildings to provide a uniform coverage that would not otherwise be possible in complex electromagnetic environments. While the cables do act as antennas, there are undesirable characteristics such as high insertion loss and larger cable loss per unit length than conventional coaxial cable. However, leaky coaxial cables do present several design aspects that are well suited for the application of detecting PEDs onboard aircraft, such as a fairly uniform coverage across all common commercial bands and unobtrusive system design. The system design presented here tries to minimize the undesirable effects by shorting cable runs to $100 \mathrm{ft}$ and placing the receiver in the center to minimize cable losses. While taking full advantage of the desirable characteristics such as uniform coverage over multiple frequency bands.

Two types of leaky coaxial cable were tested for this paper. First, Radiax ${ }^{\circledR}$ Cable (Figure 2) provided by Andrews (C) and second, FlexRad (Figure 3) provided by Times Cable ( $)$. Table 1 compares some of the physical characteristics of the different cables tested. 


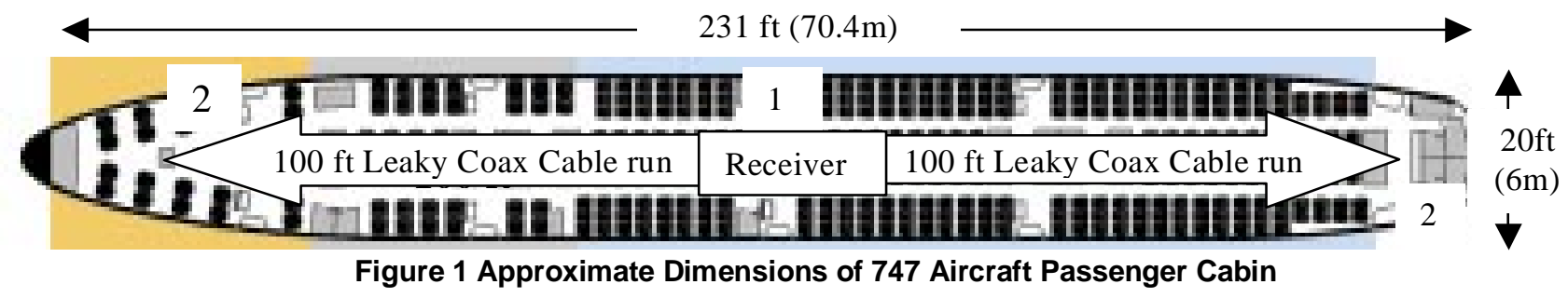

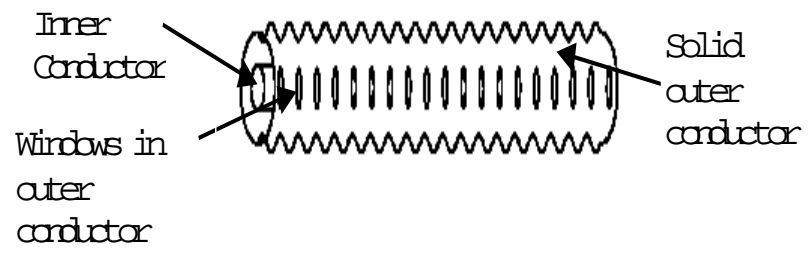

Figure 2 Diagram of Radiax Cable

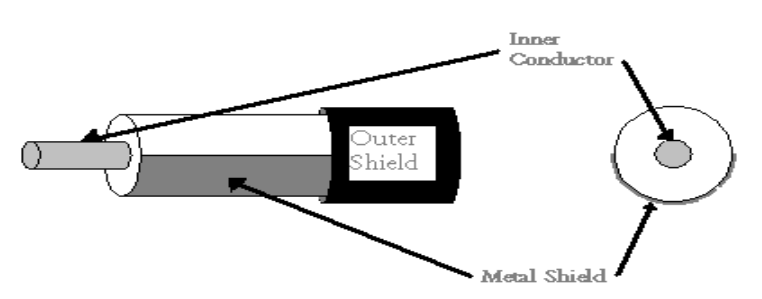

Figure 3 Diagram of FlexRad Cable

Table 1. Radiax Cable Specifications

\begin{tabular}{|c|c|c|c|}
\hline & $\begin{array}{c}\text { Andrews } \\
\text { RXL4.5- } \\
\text { 1AX } \\
\text { (Cable 1) }\end{array}$ & $\begin{array}{c}\text { Andrews } \\
\text { RXL1- } \\
\text { 1A } \\
\text { (Cable 2) }\end{array}$ & $\begin{array}{c}\text { Times } \\
\text { Flex-Rad } \\
600 \\
\text { (Cable 3) }\end{array}$ \\
\hline $\begin{array}{c}\text { Diameter } \\
\text { (in) }\end{array}$ & 0.865 in & 0.30 in & 0.52 \\
\hline $\begin{array}{c}\text { Weight } \\
\text { (lb/ft) }\end{array}$ & 0.15 & 0.055 & 0.09 \\
\hline
\end{tabular}

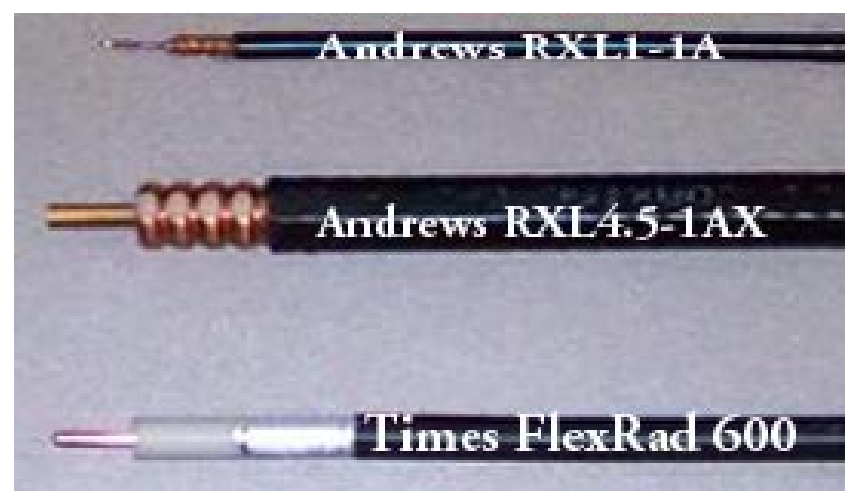

Figure 4 Cable Comparisons

\section{System Design}

The 747 aircraft fuselage was used as a design model due to a large number in service and it is representative of a wide body type commercial aircraft. Large aircraft present the most difficult design constraints for a distributed PED detection system due to increased cable loss, free space loss and, receiver complexity. For obvious reasons there will need to be slight design modifications depending on the exact model of aircraft the system is installed in, but the electromagnetic characteristics should remain approximately constant due to similar geometry of the different airframes. Approximate dimensions for the main passenger cabin of a 747-400 aircraft are shown in Figure 1.

These dimensions allow for an efficient design consisting of a single leaky coaxial cable run along the length of the passenger cabin. A single cable run minimizes weight and installation time, while also reducing the complexity of installation. Due to the electromagnetic characteristics of the passenger cabin, it will be shown that the physical location of the cable will have little effect on system performance. Using two $100 \mathrm{ft}$ sections of cable, and placing the receiver in the middle gives the two extremes shown in Figure 1.

Location 1: Passenger closest to the receiver (Prevention of false alarms): The desired system response will allow this person to use an unintentional transmitter without triggering an alarm. Due to this requirement the minimum sensitivity of the receiver will be set according to the specifications given in part 15.109 (Figure 5) of the FCC regulations for unintentionally transmitting devices. Additionally, the system needs to be dynamic enough such that a received signal of several $\mathrm{dBm}$ will not cause adverse effects such as amplifier damage.

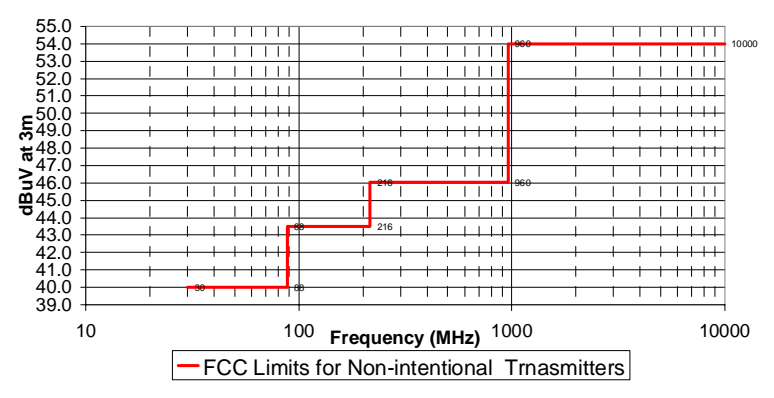

Figure 5 FCC limits for Non-intentional Transmitters

Location 2: Passenger farthest from the receiver (Detection of unauthorized transmitters): Desired system response is to detect any intentional transmitter (Assumed to be $\geq 0 \mathrm{dBm}$ ). At location 2, the attenuation 


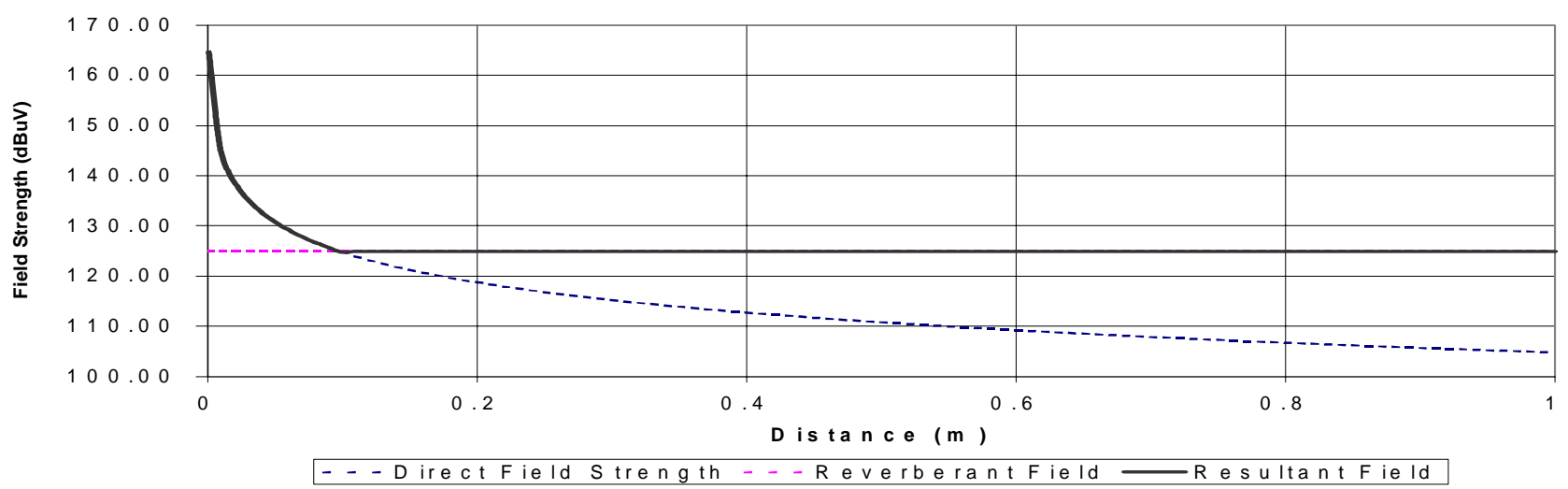

Figure 6 Field Strength Inside Passenger Cabin of 707 Aircraft

from cable loss, and the free space distance between the transmitter and the cable are both at their maximum values, resulting in the lowest received signal strength at the cable, and the largest cable loss, resulting in the lowest received power for an intentional transmitter that must be detected.

\section{Estimated Field Strength}

To determine the distance where the dominant mode of coupling switches from direct coupling to a reverberant field, it is necessary to simulate the electromagnetic environment of the passenger cabin. This was done by comparing the power density inside the reverberation chamber with the average power density collected by Naval Surface Warfare Center (NSWC) [5] inside the passenger cabin of a 707-720B aircraft (The passenger cabin of the aircraft tested was essentially intact, but stripped of all seats). The presence of 200 seats in the hull of an aircraft will reduce the average cavity gain by about $1.26 \mathrm{~dB}$ at 100 $\mathrm{MHz}$ and $0.89 \mathrm{~dB}$ at $5.8 \mathrm{GHz}$ [7] therefore, seating configuration will have little effect on the chamber characteristics.

Using Equation 1 [9], power transmitted is converted to electric field strength assuming an isotropic radiator and no reverberant effects. Then using the average power density $\left(-52 \mathrm{dBm} / \mathrm{cm}^{2}\right)$ and Equation 2 [8], the estimated reverberant field strength of the aircraft was superimposed on the graph resulting in Figure 6, where the reverberant field is dominant greater than 0.1 meters from the transmitter.

$$
\begin{aligned}
& P_{P R P}=\frac{E^{2} \cdot 4 \pi \cdot R^{2}}{120 \cdot \pi} \Rightarrow \\
& E=\sqrt{\frac{30 \cdot P}{R^{2}}}
\end{aligned}
$$

$\mathrm{P}_{\mathrm{PRP}}=$ Equivalent peak radiated power $(\mathrm{W})$

$\mathrm{E}=$ Electric field intensity $(\mathrm{V} / \mathrm{m})$

$\mathrm{R}=$ Distance in meters from source

$$
\begin{aligned}
& E_{E S T}=\left\langle\frac{8 \cdot \pi}{\lambda} \cdot \sqrt{5 \cdot \frac{\mathrm{P}_{\text {MaxReceived }}}{\eta_{\mathrm{rx}}}}\right\rangle \\
& E_{E S T}=\text { FieldStrength }(\mathrm{V} / \mathrm{m}) \\
& \lambda=\text { WaveLength } \\
& \eta_{\mathrm{rx}}=\text { AntennaEfficiency } \\
& \mathrm{P}_{\text {Max Received }}=\text { MaximumPower Received }
\end{aligned}
$$

Using time averaging, the data in Figure 7 was collected inside the reverberation chamber. This shows the insertion loss inside a reverberant field across frequency with mechanical stirring. While the power density is not shown, Figure 7 does show the uniform insertion loss into the cable. The precise insertion loss is determined using the data collected inside the semi-anechoic chamber where the electric field is easily calculated.

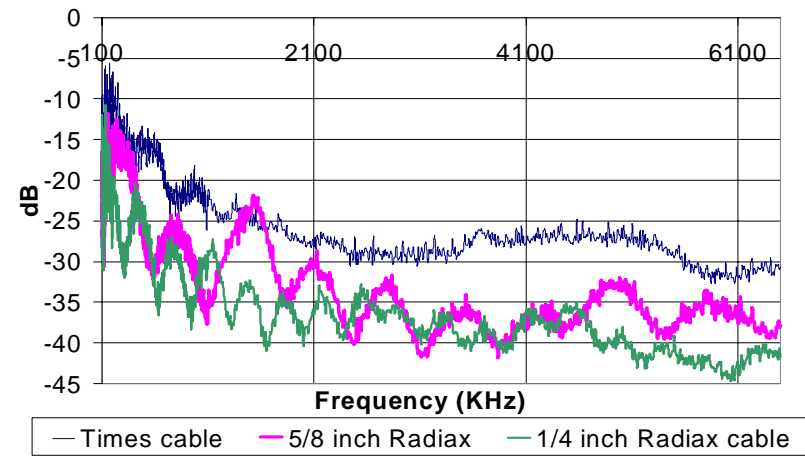

Figure 7 Reverberation Chamber received Power

An additional property investigated in the reverberation chamber was the minimum distance from a metal surface where the leaky coaxial cable is effective as an antenna. This was determined by measuring the cable loss (Figure 8) and insertion loss (Figure 9) at several heights from the metal floor using a 15-foot segment of cable. 


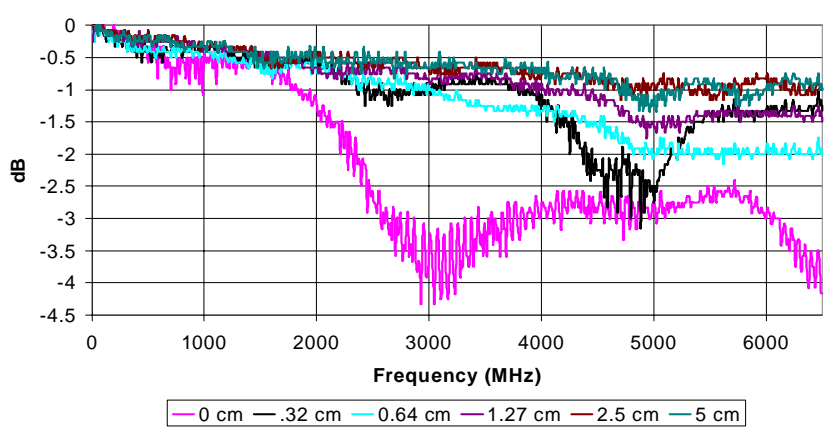

Figure 8 Cable Loss Vs. Height From Metal Surface

The cable loss is measured by connecting one end of the leaky coaxial cable to a spectrum analyzer and the other end to a signal generator. The cable loss is then the system throughput loss. Insertion loss is measured by terminating one end of the leaky coaxial cable and radiating a signal to determine the level received by the spectrum analyzer through the cable. Concluded from these two tests is that the cable performs uniformly at distances greater than 2.5 $\mathrm{cm}$ from a metal surface.

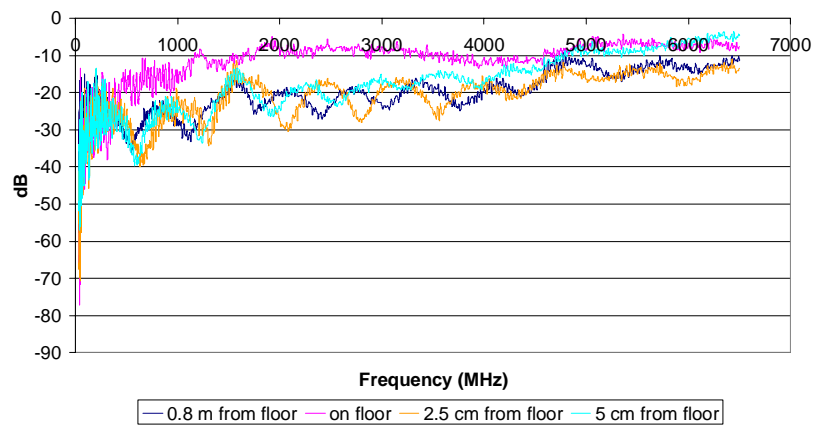

Figure 9 Insertion Losses inside Reverberation
Chamber at Various Heights from Floor

Once the effects of the reverberation chamber were recorded the leaky coaxial cable was placed in a semianechoic chamber to determine the insertion loss into the cable. Using calibration data provided by the manufacturer, the antenna's field strength $(\mathrm{dB} \mu \mathrm{V} / \mathrm{m})$ at the cable was determined for a distance of 1 and 3 meters. Once the field strength present outside the cable is known, the insertion loss into the cable in $\mathrm{dB}$ can be determined using Equation 3. Figure 10 shows the insertion loss for various locations along the cable as a function of distance from the cable termination.

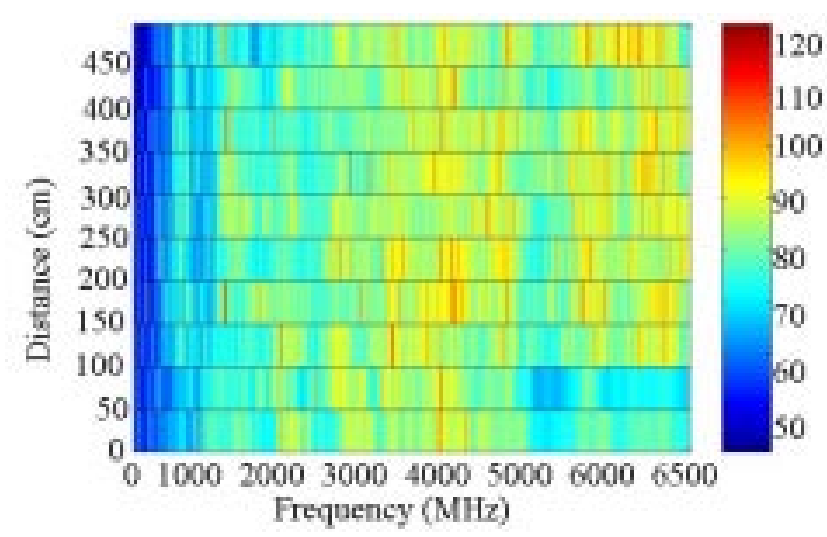

Figure 10 Semi-Anechoic Chamber Insertion Losses

$$
V_{\text {Received }}(d B \mu V)=V_{\substack{\text { Incident } \\ \text { field }}}(d B \mu V / m)-V_{\substack{\text { Coupling } \\ \text { Factor }}}(1 / m)
$$

This gives a direct correlation of field strength $(\mathrm{dB} \mu \mathrm{V} / \mathrm{m})$ outside the cable to received voltage $(\mathrm{dB} \mu \mathrm{V})$ at the terminal of the Leaky coaxial cable and is not dependent on the distance of the transmitter from the cable. Instead, it is a property of the cable itself.

\section{Broadband Frequency Coverage and Null Patterns}

In order to compare mode stirred reverberation chamber field strength data with airplane measurements data, it needed to be determined if there will be sufficient modal stirring provided in the passenger cabin to justify using mechanical stirrers while collecting data in the reverberation chamber. While there are not mechanical stirrers onboard an aircraft, several other sources of modal stirring occur, such as flexing of the aircraft's hull while in flight, and relative movement between the transmitting antenna and the aircraft will provide some stirring.

To investigate if relative movement between the antenna and the reverberation chamber boundaries provided a corresponding shift in the null pattern received by the spectrum analyzer, the leaky coaxial cable was placed in the reverberation chamber and the transmitting antenna was moved around a small area showing the development of the nulls for different antenna locations without mechanical stirring. As the antenna was moved, it became obvious that the pattern of nulls also shifted. Additionally, this phenomenon is observed in data collected inside the semi-anechoic chamber. Where by collecting data at several locations along the length of the cable, Figure 10 show that the nulls present at the spectrum analyzer are not stationary but are dependent on the location where the signal is coupled into the cable. The combination of the two results provides confidence that passenger movement and airplane flexure will remove nulls received by a detection system as a function of time averaging and therefore justifies the use of mechanical 
stirring while inside the reverberation chamber to average out nulls.

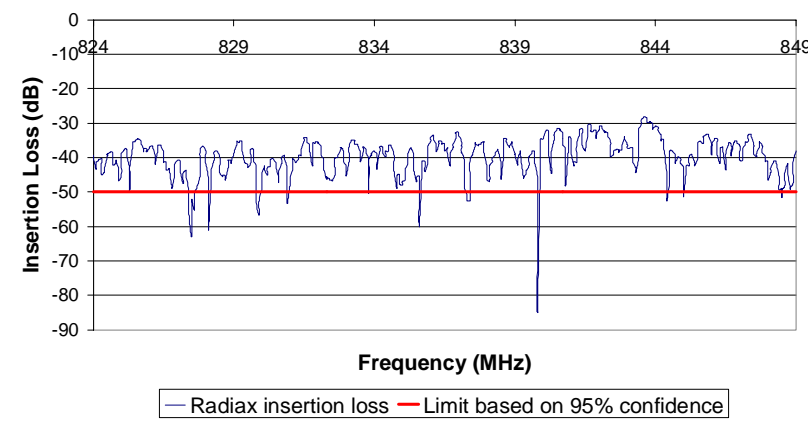

Figure 11 Cellular Band Null Pattern Corrected for Airplane Losses

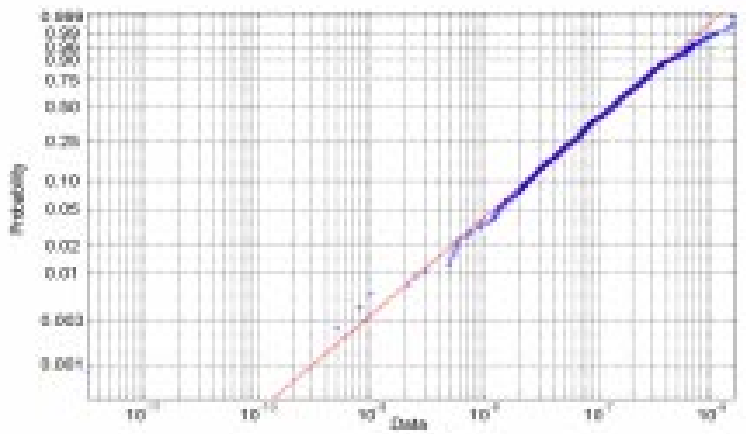

Figure 12 Weibull Probability Distribution for Cellular Telephone Band

To ensure system feasibility even if there is not sufficient stirring provided onboard the aircraft, Figure 11 shows a plot of the cellular telephone band without mechanical stirring. This shows the deep nulls that develop without modal stirring. Additionally, Figure 12 shows a Weibull cumulative distribution function, which was used to determine the $95 \%$ confidence level without stirring. With these findings it was determined that even if there is not sufficient stirring provided onboard the aircraft, that the system design would still be sound with a $95 \%$ confidence level (shown as the red line in Figure 11).

\section{Detector Design}

While a superhetrodyne receiver would be a good choice due uniform coverage across a wide frequency range, the proposed receiver instead uses multiple filters to continuously monitor all frequency bands. This design decision is based on the requirement of the superhetrodyne receiver requiring a local oscillator. While a receiver system could be designed to meet the FCC requirements for non-intentional transmitters, it is thought that a passive system will present advantages during certification for onboard use. The link budge is calculated based on the use of multiple filter circuits.

\section{Link Budget}

The power received is dependent on several factors such as power transmitted by the PED, insertion loss of the leaky coaxial cable and, free space loss at the particular frequency. From Figure 6 it is seen that the free space distance only effects field strength at distances of less than $0.1 \mathrm{~m}$. Figure 13 shows the actual received device levels inside the semi-anechoic chamber. Using these values, Table 2 shows the estimated insertion losses for actual devices in the frequency bands of interest.

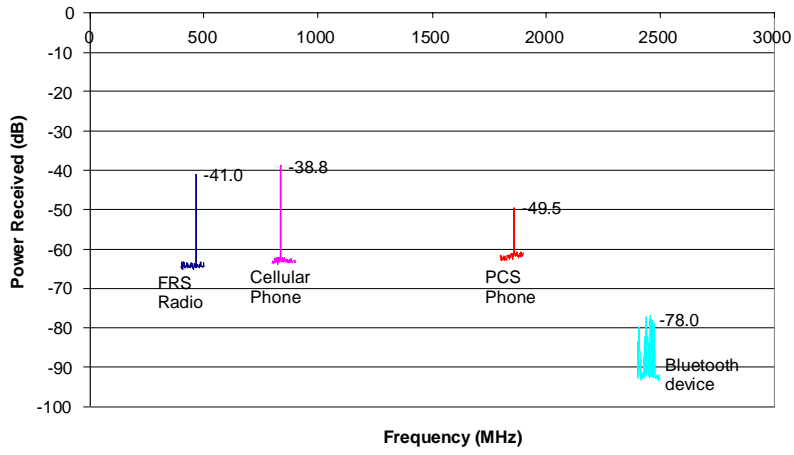

Figure 13 Device Signatures for Frequency Bands of Concern

Table 2 Received signal strength for actual PED devices using 5/8" Radiax Cable at $1 \mathrm{~m}$

\begin{tabular}{|c|c|c|c|}
\hline Frequency Band & $\begin{array}{c}\text { Approximate } \\
\text { power } \\
\text { transmitted } \\
(\mathrm{dBm})\end{array}$ & $\begin{array}{c}\text { Power } \\
\text { Received } \\
(\mathrm{dBm})\end{array}$ & $\begin{array}{c}\text { Insertion } \\
\text { losses } \\
(\mathrm{dB})\end{array}$ \\
\hline $\begin{array}{c}\text { FRS Radio } \\
(\approx 460 \mathrm{MHz})\end{array}$ & 27 & -41 & 68 \\
\hline $\begin{array}{c}\text { Cellular } \\
(\approx 830 \mathrm{MHz})\end{array}$ & 27 & -39 & 66 \\
\hline $\begin{array}{c}\text { PCS } \\
(\approx 1860 \mathrm{MHz})\end{array}$ & 27 & -50 & 77 \\
\hline $\begin{array}{c}\text { Bluetooth } \\
(\approx 2400 \mathrm{MHz})\end{array}$ & 0 & -78 & 78 \\
\hline
\end{tabular}

From Table 2, the limiting received power is for a Bluetooth device. Due to the desire to cover several frequency bands using the same antenna there will be additional losses associated with a power divider depending on the number of separate bands covered. To cover the frequency bands listed in the table for instance a 1:4 power splitter will be required. The loss associated with the splitter is an additional $6 \mathrm{~dB}$ bringing the true received power to approximately $-84 \mathrm{dBm}$. This will require a preamplifier to boost the signal strength by $30-40 \mathrm{~dB}$, which will allow the signal to be sent to a power meter with a tangential signal sensitivity (TSS) of approximately -50 
$\mathrm{dBm}$. The output of the power meter is a small DC voltage $(\approx 10 \mathrm{mV})$. Since the output of the power meter is proportional to the input power the signal can drive an instrumentation amplifier with the output of the instrument amplifier connected to a comparator and finally an alarm circuit.

\section{Noise Analysis}

To determine the noise floor of the system several assumptions were needed.

1. The Bluetooth band is used as the limiting case for noise. This is due to the Bluetooth band's wide frequency band of 2.4 to $2.5 \mathrm{GHz}$. To cover the entire frequency band, a band pass filter with a bandwidth of $100 \mathrm{MHz}$ will be required.

2. An amplifier with an assumed noise factor (NF) of 4.0 is used.

3. The transmission line loss is assumed from the base of the leaky coaxial cable to the receiver. This assumption follows from the way insertion loss was found to be at the termination of the leaky coaxial cable and can therefore be assumed as small (@1 dB).

4. Due to the large noise factor of the amplifier, the antenna noise temperature will not have a large effect on the system. To compensate for the antenna noise temperature, the final result was rounded up.

To calculate the input noise floor, the following 3 equations from Reference Data for Engineers [10] was used.

$$
\begin{aligned}
& \mathrm{T}_{\mathrm{E}}=\mathrm{T}_{\mathrm{A}}+(\mathrm{L} \cdot \mathrm{F}-1) \cdot \mathrm{T}_{0} \\
& \mathrm{~T}_{\mathrm{E}}=\text { Effective Noise Temperatue of System } \\
& \mathrm{T}_{\mathrm{A}}=\text { Antenna Noise Temperatue } \\
& \mathrm{L}=\text { Transmission Line Loss } \\
& \mathrm{F}=\text { Noise Factor of Receiver } \\
& \mathrm{T}_{\mathrm{o}}=290 \mathrm{~K} \\
& \mathrm{~N}_{\mathrm{i}}=\mathrm{kBT}_{\mathrm{E}} \\
& \mathrm{dBm}_{\mathrm{i}}=-198.6+10 \log (B)+10 \log \left(T_{E}\right) \\
& k=1.38 \times 10^{-23} \\
& B=\text { Bandwidth in Hz } \\
& \mathrm{T}_{\mathrm{E}}=\text { Effective noise temperatu re }
\end{aligned}
$$

Using Equation $4, \mathrm{~T}_{\mathrm{E}}$ is calculated to be approximately $450 \mathrm{~K}$. Then using Equations 5 and 6, the noise floor is calculated to be $-92 \mathrm{dBm}_{1}$. Since the received signal for a Bluetooth device is estimated to be at $-84 \mathrm{dBm}$, the signal will appear slightly above the noise floor.

\section{Conclusion}

Based on data collected, leaky coaxial cable is capable of performing as a distributed PED detector onboard an aircraft across all frequency bands of interest. Due to the limitations of the reverberation chambers the data below $100 \mathrm{MHz}$ will need to be verified during further testing.

While the data shows that the environment inside the aircraft is reverberant and, therefore, could be monitored as efficiently with a single antenna. The uniform field is not consistent throughout the length of the aircraft due to the many apertures (windows) in the hull and walls dividing the sections. This is overcome with the leaky coaxial system described since the signal decays in a predictable fashion once coupled onto the cable. Several other factors are unaccounted for in this analysis that are present in a real environment such as passengers and other chamber loads. These loads will result in an overall lower Q value for the passenger cabin.

Due to the space constraints onboard an aircraft the leaky coaxial cable allows for an antenna the size of the cable that would otherwise be used to carry the signal back from another system. While the cable does not perform particularly well over any one frequency band it was able to cover a frequency range that required three conventional antennas to produce the test signal, thus providing a good compromise between space required and overall system performance.

\section{References}

[1] De Donno, Fabio, Capt., Seventh Flight Safety Conference, "Portable Electronic Devices on Aeroplanes", 2000

[2] FAA AC 21, www.faa.gov/avr/afs/acs/ac91211a.pdf

[3] FCC 14CFR15 section 22 http://www.fcc.gov/oet/info/rules/

[4] FCC CFR 22 section 917 , http://www.access.gpo.gov/nara/cfr/waisidx_02/47cfr22_02.html

[5] Hatfield, Michael O. Naval Surface Warfare Center, Dahlgren, "Phase II Demonstration Test of the Electromagnetic Reverberation Characteristics of a Large Transport Aircraft", NSWCDD/TR-97/84

[6] Ladbury, John. "Evaluation of the NASA LARC Mode-Stirred Chamber Facility”, NIST, January 1999

[7] Nguyen, Truong. "RF Loading Effects of Aircraft Seats in an Electromagnetic Reverberating Environment", NASA Langley Research Center Hampton Virginia, NASA TM-2000-21062

[8] RCTA DO-160D Section 20, "Environmental Conditions and Test Procedures for Airborne Equipment”, Prepared by SC-135, July 29, 1997

[9] RCTA DO-233, "Portable Electronic Devices Carried Onboard Aircraft", Prepared by SC-177, 20 August 1996

[10] Reference Data for Engineers, Eight Edition, SAMS publishing 1993 